\title{
How human is your computer? Measuring ethopoeic perceptions of computers
}

\author{
J. P. Charlton \\ Department of Psychology and Life Sciences, University of Bolton, UK
}

\begin{abstract}
The extent to which people have human-like perceptions of, and relationships with, computers is considered along with previous attempts at measuring these phenomena. A distinction is made between the unrealistic notion that adults anthropomorphise the computers that they use everyday in the same way that they anthropomorphise entities such as animals (e.g. ascribe feelings and purpose to them) and the more realistic idea that some people perceive such computers as having human-like characteristics while not believing that they are sentient and purposeful (following previous work, such perceptions are labelled ethopoeic). Based upon the assumption that one way of assessing the extent of people's ethopoeic perceptions of computers is to measure the extent to which they consider that words describing human-like cognitive and volitional qualities can also be applied to computers, a short scale measuring ethopoeic perceptions of computers is developed. This scale, entitled the Computer Perceptions Scale (CPS), is shown to be unifactorial and reliable. CPS scores are shown to be negatively related to computing experience and trait computer anger. Finally, a research agenda is outlined in which it is planned to use the scale to investigate the extent to which individual differences in ethopoeic perceptions of computers are involved in both the differential attribution of blame to computers for anger inducing computing incidents and the degree to which the anger resulting from such incidents causes overt expression of anger towards computers.

Keywords: human-computer relationships, anthropomorphism, ethopoeia, individual differences, human sex differences, computer experience, anger.
\end{abstract}

\section{Introduction}

\subsection{The nature of human-computer relationships}

The idea that people have social relationships with computers has been around for a long time, Turkle [1] perhaps being the most prominent early proponent of 
this idea. Much of the empirical work in this area has been conducted by Nass and colleagues. These authors' experimental studies have shown that in many respects people interact with computers in the same way as they do with humans. For example, among other things, people see computers which positively evaluate themselves but negatively evaluate other computers as less friendly than computers making the opposite types of evaluation, also people gender stereotype and ethnically stereotype computers, reciprocate accordingly when computers help or fail to help them, show self-serving attribution bias with respect to computers, and show politeness to computers (see Nass \& Moon [2] and Reeves \& Nass [3] for detailed summaries of Nass's experiments).

Recent work by Sundar [4] pursued the possibility that computer anthropomorphism may be responsible for social behaviours of the type observed in Nass's studies. Here it was shown that people exhibit "loyalty" to computers by limiting their usage of computer terminals to just a few terminals within a computer laboratory. However, this behaviour could not be unambiguously attributed to anthropomorphism since scores on a questionnaire considered to measure anthropomorphism and scores on a behavioural consistency questionnaire were both predictive of selective terminal usage.

A final example of work in this area is that of Hall and Cooper [5]. This looked at individual differences in the extent to which people use tool-like or human-like references when using computers. Here, people were asked to write essays describing unsuccessful and successful computing experiences. Judges were asked to rate the resulting essays in terms of the extent to which the computer was referred to in mechanical-like or human-like terms. Among other things, one major finding was that, irrespective of whether the incident involved success or failure, males had a greater tendency to give human-like descriptions. Also, there was a significant sex by computing experience interaction whereby females with greater computing experience gave more mechanical descriptions but males with greater computing experience gave more human-like descriptions. For males, these findings were explained in terms of greater experience resulting in a greater sense of control, previous research being cited as showing that people tend to view important objects in a more personalized manner when they perceive themselves to have control and mastery over these objects. The fact that females did not do this was explained by citing previous studies showing that females use and value non-verbal cues more when communicating than males do, and therefore the absence of such cues from the computer results in females' interactions with computers being more impersonal, with feedback from the computer therefore being less likely to be perceived as affirming mastery and control. In reviewing these results, Huff et al. [6] note that males appeared to use a different type of language to describe their interactions with computers, which reflected a closer personal relationship with computers, rather than having a real belief that computers possess human-like attributes.

Although previous authors (e.g. $[1,4,7]$ ) have used the term anthropomorphism in connection with computers, neither the work of Sundar nor that of Hall and Cooper constitutes evidence that people anthropomorphise computers, and in fact Nass and Moon [2] ascribe people's readiness to respond in the same 
way to computers as they do to people to mindlessness rather than anthropomorphism.

Anthropomorphism is "the attribution of a human form or personality to a god, animal or thing" [8, p.45], and, to paraphrase Kennedy [9], entails the belief that a non-human entity has feelings and purposes and acts upon them. While few, if any, adults are likely to have such beliefs in respect of commonplace computers [2], people do often respond to computers as though they were human while at the same time realising that computers do not merit such responses. Nass and Moon [2] term this ethopoeia (from the Greek ethos meaning character, and poeia meaning representation [10]). Ethopoeia, and the mindlessness in responding to computers that characterises it, is said to result from certain human-like cues from the computer triggering scripts that usually would only be appropriate for human-human interaction. Scripts are deeply rooted representations of knowledge of the behaviours that apply in a given set of circumstances and that steer our actions when we encounter such circumstances [11]. Cues responsible for triggering inappropriate scripts when computing include the interactive nature of human-computer communication, the use of words in human-computer dialog, and the fact that computers take-on roles previously carried out by humans [2]. Also, the fact that we use human-like terminology in describing computers may be implicated. For example, we use terms describing human intellectual functioning such as memory, reading and writing $[5,12]$, and we talk in terms of computers being friendly [12]. The rationale for the development of the instrument recounted in this paper assumed that the activation of scripts because of the aforementioned reasons can lead people to conceive of computers as having human-like properties such as cooperativeness, sociableness and trustworthiness, and that computers' ability to perform complex tasks quickly, and produce the correct results, can make people credit computers with cognitive characteristics similar to those that they would credit a human performing in the same manner with.

\subsection{Measuring human-like perceptions of computers}

To summarise the above, although people are unlikely to anthropomorphise computers, there is reasonable agreement that people have human-like relationships with them, that people sometimes respond as though computers have human-like characteristics, and that people employ human-like language when describing computers. The term ethopoeia has been used in relation to these responses to computers. Towards the end of studying the role of ethopoeic perceptions of computers in the genesis and expression of computer-related anger, the work described presently sought to develop and test a short measure of the extent to which people have ethopoeic perceptions of computers. A brief review of the methods used to collect data concerning people's perceptions of, and relationships with, computers in the previously mentioned studies will make it clear why it was necessary to devise such an instrument.

Hall and Cooper [5] used the rating of essays on a five-point scale for the extent to which human-like references (e.g. the computer “....accepted my program and assured me" p.53) or machine-like references (e.g. “...this should 
register into the computer" p.53) characterised the essay. While such a procedure is useful in considering the nature of people's relationships with computers, for reasonably large sample sizes it is labour intensive. Sundar [4] adopted a more readily usable questionnaire methodology, creating 12 items based upon suggestions from the book by Reeves and Nass [3] as to how designers might make computer interfaces more socially-oriented. Four of these items read...

"I would be less impatient if a slow-loading computer apologized for the amount of time it was taking"

"When I log out, I would be pleased if the computer thanked me and wished me good day"

"I would be glad if a computer praised my job"

"If the computer does not perform the function I ask, I will curse it"

(Sundar [4] p.112)

Along with most other items in the questionnaire, these items appear to be largely restricted to measuring people's desires for computers to communicate with them and people's tendencies to attempt to communicate verbally with computers. Although such desires and tendencies may reflect aspects of ethopoeia, this questionnaire does not focus directly upon ethopoeic perceptions of computers, and was therefore considered unsuitable for examining the role of such perceptions in our planned studies of computing anger. Finally, Nass, Lombard et al. [7] also used a questionnaire method, asking people whether they thought that there were presently "...computers or things that use or contain computers..." that could exhibit characteristics such as anger, annoyance, shame and embarrassment. From its description this questionnaire mainly appears to measure emotional ethopoeic perceptions concerning state of the art computing technology, and, as discussed above, it is likely that only a tiny minority of adults, if that, would express any agreement with the idea that the computers they interact with on a daily basis are capable of such feelings.

\section{The development of the Computer Perceptions Scale}

Given that none of the previously mentioned studies had developed a psychometric measure of ethopoeic perceptions of commonly used computers, the present research set out to develop such a measure and to provide some preliminary evidence concerning its properties. In reporting the development of this measure it is hoped that the instrument may prove useful to other researchers seeking to study ethopoeic phenomena. For the reasons mentioned previously, the instrument, entitled The Computer Perceptions Scale (CPS), was restricted to measuring perceptions of computers' cognitive and volitional characteristics such as intelligence and cooperativeness, and did not include perceptions of emotional characteristics.

In developing the CPS the aim was to construct a scale consisting of adjective pairs which are descriptive of people but not of tools so as to avoid ambiguity in the meaning of people's responses on the scale. Development of the scale began by searching a dictionary and thesaurus for suitable adjectives and their antonyms, account was also taken of terms mentioned in the previous literature. 
This resulted in a pilot development instrument which contained 24 adjective pairs. This was then given to two judges who were asked to rate the extent to which the pairs of words are relevant in describing tools and people. If they thought that a pair of words was completely irrelevant in describing the entity concerned (tools or people), a rating of 1 was given, a rating of 4 was given for moderate relevance, and a rating of 7 for extreme relevance, with the numbers between being used to represent points between the three above mentioned degrees of relevance. It was emphasised that the questionnaire was not asking whether the judges thought that entities possessed the qualities indicated by the adjective pairs, but rather whether they thought it appropriate to think of tools and people in terms of the adjective pair (e.g. friendly and unfriendly).

Once the two judges had rated the adjective pairs, a selection rule was adopted whereby both judges had to agree that an adjective pair was at least moderately applicable to people (a rating of 4 greater or greater), but less than moderately applicable to tools (a rating lower than 4). Nine adjective pairs were found to meet this criterion. As a final check on the validity of the items, a third judge was then asked to perform the above rating exercise on the nine items. For all nine items the ratings of this judge verified those of the previous two judges. It is worth noting that across the three judges involved in the rating exercises used to construct the scale, mean ratings for all nine of the final scored items were greater than six with respect to people (i.e. there was near consensus in terms of the adjective pairs being extremely relevant to people), and lower than two with respect to tools (i.e. there was near consensus in terms of the adjective pairs being not at all relevant to tools).

The final scale consists of 16 items: the nine scored adjective pairs shown in Table 1 in the next section and seven filler pairs selected from those in the development pool (these latter pairs are Unfriendly / Friendly, Reliable / Unreliable, Efficient / Inefficient, Helpful / Unhelpful, Temperamental / Untemperamental, Undependable / Dependable, and Hostile / Non-hostile). People are asked to rate the extent to which they think it is reasonable to describe computers in terms of the 16 adjective pairs, with the rating scheme being the same as that employed during the scale's development. Likewise, emphasis is again placed upon the fact that the questionnaire is not asking whether people think that computers actually possess the qualities described by the adjective pairs, only whether it is considered appropriate to think of computers in such terms. In deriving a scale total, responses to the nine scored adjective pairs are summated, resulting in totals ranging from a possible minimum of 9 to a possible maximum of 63 , with higher scores indicating greater ethopoeia. A copy of the scale as presented to respondents is available from the author.

\section{The psychometric properties of the CPS and some initial analyses of CPS scores' relationships with other variables}

\subsection{The factor structure and reliability of the CPS}

To investigate the psychometric properties of the CPS, 95 adult members of the general public, recruited using opportunity sampling, were asked to complete it. 
The sample consisted of 24 males (age range $=18$ to 70 years, mean age $=40.46$ years, $S D=15.72$ years) and 71 females (age range $=18$ to 62 years, mean age $=37.11$ years, $S D=11.98$ years, 1 age unknown). All respondents were computer users and the British Psychological Society's ethical principles were adhered to throughout.

A scree plot associated with an initial principal components analysis of the nine scored items indicated a unifactorial factor structure, and subsequent principal axis factoring (PAF) showed that this factor accounted for $40 \%$ of item variance. The factor matrix loadings from the PAF are given in Table 1.

Table 1: $\quad$ Factor matrix loadings for the nine scored CPS items.

\begin{tabular}{|l|c|}
\hline \multicolumn{1}{|c|}{ Item } & Loading \\
\hline Incompetent / Competent & .822 \\
\hline Cooperative / Uncooperative & .714 \\
\hline Unintelligent / Intelligent & .690 \\
\hline Untrustworthy / Trustworthy & .647 \\
\hline Stupid / Not Stupid & .638 \\
\hline Unwelcoming / Welcoming & .597 \\
\hline Authoritative / Unauthoritative & .565 \\
\hline Non-Aggressive / Aggressive & .460 \\
\hline Sociable / Unsociable & .456 \\
\hline
\end{tabular}

For the above data set, internal consistency of the scale was found to be acceptable (Cronbach's alpha $=.85$ ), and the mean score on the scale was 28.68, with an $S D$ of 11.85 and minimum and maximum observed scores of 9 and 56 respectively. Assessment of test-retest reliability on a provisional small 23 member subsample of the above sample with a three week interval between administrations showed that responses to the instrument were temporally stable with Pearson's $r(21)=.852, p<.0005$ one-tailed.

\subsection{Relationships with demographic and miscellaneous computing variables}

The CPS was also used to collect data on age, sex and computing experience in terms of approximate number of years computing experience. There was a nonsignificant positive correlation between age and CPS scores, $r(92)=.135$, $p=.195$ two-tailed. An independent samples $t$-test showed that there was no sex difference in CPS scores (male mean $=27.46, S D=11.02, n=24$; female mean $=29.10, S D=12.17, n=71), t(93)=0.58, p=.561$ two-tailed, and the effect size was minimal (Cohen's $d=.14$ ). However, a Pearson's $r$ analysis showed a significant negative relationship between CPS scores and number of years computing experience, $r(92)=-.255, p=.013$ two-tailed, with ethopoeia decreasing with experience. This approaches a medium sized effect $(r=.30)$ with respect to Cohen's [13] effect size benchmarks. Bearing in mind Hall and Cooper's [5] observations, Pearson's $r$ analyses were also computed between CPS scores and number of years computing experience for males and females separately. These analyses revealed marginally non-significant negative 
relationships for both sexes. For males $r(22)=-.363, p=.081$ two-tailed, and for females $r(68)=-.255, p=.061$ two-tailed. Although, non-significant under twotailed hypotheses, again these coefficients are both of a size approximating to Cohen's medium effect size classification.

To provide some initial information on the relationship between CPS scores and other measures of computing-related individual differences, at the same time as completing the CPS a subset of the above respondents were also asked to complete the 71 item Computing Orientations Inventory (COI). This inventory combines instruments developed in previous work by the present author $[14,15,16,17]$. The COI, which uses a five-point "strongly disagree" to "strongly agree" Likert scale response format to statements concerning computers, contains subscales measuring computer-specific anger, engagement, addiction, need for control, autonomy and anxiety. The subsample of 53 participants completing the COI consisted of 14 males (mean age $=41.86$ years, $S D=16.81$ years) and 39 females (mean age $=36.62$ years, $S D=12.40$ years).

Table 2: Pearson's $r$ coefficients for relationships between CPS and COI scores.

\begin{tabular}{|l|c|c|}
\hline \multicolumn{1}{|c|}{ COI Subscale } & $r$ & $p$ (two-tailed) \\
\hline Anger & -.299 & .030 \\
\hline Engagement & .053 & .708 \\
\hline Anxiety & -.011 & .939 \\
\hline Autonomy & -.116 & .406 \\
\hline Addiction & .095 & .497 \\
\hline Need for Control & -.030 & .832 \\
\hline
\end{tabular}

Pearson's $r$ analyses (see Table 2) revealed that only COI anger subscale scores were significantly related to CPS scores, with trait computing anger decreasing as ethopoeia increased. This coefficient represented a medium effect size. Of the other coefficients, only that for autonomy was of a size which Cohen [13] deems worthy of describing as a small effect $(r=.10)$.

\section{Discussion}

The two previous sections of this paper described the development and testing of a short instrument measuring ethopoeic perceptions of computers' cognitive and volitional characteristics. The instrument was shown to be unifactorial and to be reliable. Other analyses then provided some preliminary evidence as to the nature of the construct measured by the CPS.

Contrary to the findings of Hall and Cooper [5], but similarly to those of Nass et al. [7] concerning emotional perceptions, there was no sex difference in cognitive and volitional ethopoeic perceptions. However, contrary to Nass et al. [7], perceptions varied with experience, with less experienced people having greater ethopoeic perceptions. When sex and experience were considered jointly, sex was not shown to have a moderating effect upon the relationship 
between computing experience and CPS scores. Thus, rather than ethopoeia increasing with experience for males but decreasing with experience for females as found by Hall and Cooper, the previously mentioned negative relationship between experience and ethopoeia held for both males and females, although the low power associated with the smaller sub-samples when the data was broken down by sex resulted in marginally non-significant coefficients for both sexes. At present, it is unclear if this is because Hall and Cooper's observations lack robustness or because the CPS taps a construct that is somewhat different from that tapped by these authors' methodology. Nevertheless, the present results suggest that, irrespective of sex, more experienced people are less likely to entertain ethopoeic perceptions of computers as measured by the CPS. Whether this is because their experiences increasingly instil a perception that computers are simply machines with no or few human-like characteristics, or whether it is because people who acquire the greatest amounts of computing experience vary on other psychological dimensions is an issue for future research.

Analysis of relationships between CPS scores and scores on measures of a number of computer attitudes constructs showed that the only sizable relationship was that involving computer anger. This result is discussed briefly below in the context of outlining planned future research using the CPS.

\subsection{Computer anger and future research using the CPS}

Development of the CPS was conducted towards the end of conducting research into possible roles played by ethopoeic perceptions of computers in the generation and overt expression of computing-related anger. The research will proceed under the assumption that differences in the extent to which people are prepared to endorse the use of language containing human-like descriptors with respect to computers, as shown by the CPS, may indicate differences in cognitions about computers, with those who are prepared to use such language having closer, more human-like, relationships with computers, as reflected in the writings of Huff et al. [6].

Within the framework of the Smith and Lazarus variant of emotional appraisal theory [18], it is planned to test a number of hypotheses. First, people with greater and lesser ethopoeic tendencies are expected to be more likely to blame the computer and other people respectively for anger causing computing incidents. Second, two competing hypotheses will be examined. From one perspective it is reasonable to suggest that people with greater ethopoeic tendencies (assuming that these indicate closer human-like relationships with computers) should be more inclined to overtly express their anger towards computers when their expectations as to how computers should behave in their interactions with them are confounded [19]. However, if the idea that scripts that are normally triggered when interacting with other humans also tend to be triggered when people interact with computers [2] increasingly applies as people have more human-like relationships with computers, then ethopoeia should moderate the relationship between anger intensity and the displaying of overt anger towards the computer. That is, given the same intensity of internal anger, the greater the ethopoeia the lesser the tendency to display overt anger towards 
computers should be, since scripts which lead people to restrain themselves from overt displays of anger when interacting with humans should also be activated when interacting with computers. Since many of the items on the COI computer anger subscale tap a tendency to overtly display anger when computing, the fact that there was a negative relationship between COI anger scores and CPS scores provides some evidence in support of this idea.

To consider the above hypotheses, qualitative and quantitative data will be collected by asking people to record their thoughts and behaviours using digital voice recorders immediately after experiencing anger inducing computing incidents. In addition to using this data to evaluate the hypotheses mentioned, the data will also be used in analyses of the convergent validity of the CPS, with CPS scores being expected to be positively correlated with the extent to which people make utterances involving attributions of human-like characteristics to the computer when describing its role in anger inducing computing incidents.

\subsection{Conclusions}

The present research effort developed a short measure of ethopoeic perceptions of computers' cognitive and volitional characteristics, and provided some evidence that this instrument has acceptable psychometric qualities. Although validation of the instrument is ongoing, it appears as though people's ethopoeic perceptions of computers decrease as both their computing experience and computing anger proneness increase, however causal relationships cannot be inferred at present. The lack of floor effects found in testing the CPS shows that many people apply descriptors of human-like cognitive and volitional characteristics to computers. While this is unlikely to indicate that such people ascribe human-like feelings and purposes to computers, it is likely that there are important differences in the computer-related cognitions of people with greater and lesser tendencies to apply the aforementioned descriptors, and that these differences result in different computer-related attitudes and behaviours. One area in which it is planned to consider these issues in greater depth is that involving the role of ethopoeia-related cognitions in the generation and expression of computer-related anger.

\section{References}

[1] Turkle, S., The second self, Simon and Schuster: New York, 1984.

[2] Nass, C. \& Moon, Y., Machines and mindlessness: Social responses to computers. Journal of Social Issues, 56(1), pp.81-103, 2000.

[3] Reeves, B. \& Nass, C., The media equation: How people treat computers, television, and new media like real people and places, Cambridge University Press: Cambridge, 1996.

[4] Sundar, S.S., Loyalty to computer terminals: Is it anthropomorphism or consistency? Behaviour \& Information Technology, 23(2), pp.107-118, 2004.

[5] Hall, J. \& Cooper, J., Gender, experience and attributions to the computer. Journal of Educational Computing Research, 7(1), pp.51-60, 1991. 
[6] Huff, C.W., Fleming, J.H. \& Cooper, J., Gender differences in humancomputer interaction. In Search of Gender Free Paradigms for Computer Science Education, eds. C.D. Martin and E. Murchie-Beyma, International Society for Technology in Education: Eugene, OR, pp. 19-32, 1992.

[7] Nass, C.I., Lombard, M., Henriksen, L. \& Steuer, J., Anthropocentrism and computers. Behaviour \& Information Technology, 14(4), pp.229-238, 1995.

[8] The Concise Oxford Dictionary (8th ed.), Oxford University Press: Oxford, 1990.

[9] Kennedy, J.S., The new anthropomorphism, Cambridge University Press: Cambridge, 1992.

[10] Burton, G., Silva Rhetoricae. Brigham Young University, College of Humanities. Provo: UT, http://humanities.byu.edu/rhetoric/Figures/E/ ethopoeia.htm.

[11] Schank, R.C. \& Abelson, R., Scripts, plans, goals and understanding, Lawrence Erlbaum: Hillsdale, NJ, 1976.

[12] Marakas, G.M., Johnson, R.D. \& Palmer, J.W., A theoretical model of differential social attributions toward computing technology: When the metaphor becomes the model. International Journal of Human-Computer Studies, 52(4), pp.719-750, 2000.

[13] Cohen, J., Statistical power analysis for the behavioral sciences (2nd ed.), Lawrence Erlbaum: Hillsdale, NJ, 1988.

[14] Charlton, J.P. \& Birkett, P.E., The development and validation of the Computer Apathy and Anxiety Scale. Journal of Educational Computing Research, 13(1), pp. 41-59, 1995.

[15] Charlton, J.P., A factor-analytic investigation of computer 'addiction' and engagement. British Journal of Psychology, 93(3), pp.329-344, 2002.

[16] Charlton, J.P., Measuring perceptual and motivational facets of computer control: The development and validation of the Computing Control Scale. Computers in Human Behavior, 21(5), pp.791-815, 2005.

[17] Charlton, J.P. \& Kappas, A., Comparing appraisals of driving-related and computing-related anger. In preparation.

[18] Smith, C.A. \& Lazarus, R.S., Appraisal components, core-relational themes, and the emotions. Cognition and Emotion, 7(3/4), pp.233-269, 1993.

[19] Miller, C.A., Human-computer etiquette: Managing expectations with intentional agents. Communications of the ACM, 47(4), pp.31-34, 2004. 